\title{
The Role of Oropharyngeal Barotrauma as a Cause of Pneumomediastinum: Report of a Case*
}

\author{
Hakki Ulutas $^{1 \#}$, Erdal Yekeler ${ }^{2}$, Zafer Hasan Ali Sak ${ }^{3}$, Bayram Altuntas ${ }^{4}$ \\ ${ }^{1}$ Department of Thoracic Surgery, Faculty of Medical, University of Inonu, Malatya, Turkey; ${ }^{2}$ Department of Thoracic Surgery, \\ Ataturk Training and Research Hospital for Chest Disease and Chest Surgery, Ankara, Turkey; ${ }^{3}$ Department of Pulmonary Medicine, \\ School of Medicine, Harran University, Sanliurfa, Turkey; ${ }^{4}$ Department of Thoracic Surgery, Regional Training and Research \\ Hospital, Erzurum, Turkey. \\ Email: \#drhakkiulutas@yahoo.com
}

Received August 13 ${ }^{\text {th }}, 2012$; revised September 18 ${ }^{\text {th }}, 2012$; accepted September $28^{\text {th }}, 2012$

\begin{abstract}
Free air or gas in mediastinum is defined as pneumomediastinum. This is a rare condition which orginates from over distention of alveolus and alveolar rupture by barotrauma. A 6-year-old boy was admitted to our department with sudden onset, swelling of neck and face that developed during drinking water from tap by using his mouth. Physical examination revealed the presence of subcutaneous emphysema over the two side of the face that extended toward the neck bilaterally. The chest X-ray and CT of the thorax, when performed, revealed the diagnosis of pneumomediastinum and extrathoracic subcutaneous emphysema. Interestingly neither trachea-bronchial nor esophageal pathology was found by emergent rigid bronchoscopy and endoscopy for etiology of pneumomediastinum. Antibiotic treatment and oxygen therapy were given to the patient with chest pain and dyspnea. During the following days, the patient's condition improved notably, with almost total resolution of the cervical emphysema and pneumomediastinum confirmed by daily chest X-ray and control thorax CT. He was discharged home after 6 days. The emphysema gradually resolved. Pneumomediastinum caused by barotrauma is a rare condition and only conservative treatment is required when the other causes are ruled out.
\end{abstract}

Keywords: Oropharyngeal; Barotrauma; Pneumomediastinum

\section{Introduction}

Pneumomediastinum is the presence of air or gas in the mediastinum. This conditon is caused by intraabdominal pressure increase generally in young patients as a result of severe cough and heavy exercise [1]. Incidence of pneumomediastinum due to barotrauma among emergency department patients is $1 / 12,500-1 / 30,000$ [2,3]. In this paper, a case of oropharyngeal barotrauma-induced pneumomediastinum is presented.

\section{Case}

A 6-year-old boy was admitted to our department with sudden onset, swelling of neck and face developed during drinking water from tap by using his mouth. Physical examination revealed the presence of subcutaneous emphysema over the two side of the face that extended toward the neck bilaterally. The chest X-ray and CT of the thorax when performed revealed the diagnosis of pneu-

* Conflict of Interest Statement: Hakki Ulutas and Co-authors have no a financial relationship with the organization that sponsored the research. \#Corresponding author. momediastinum and extrathoracic subcutaneous emphysema, no additional pathology was found in lung parenchyma and mediastinum (Figures 1 and 2). Interestingly neither tracheabronchial nor esophageal pathology was found by emergent rigid bronchoscopy and endoscopy for etiology of pneumomediastinum. Patient was consultated with a laryngologist. Upper airway was normal. Antibiotic treatment and oxygen therapy were given to the patient with chest pain and dyspnea. After 48 hours, the pneumomediastinum and emphysema improved notably, with almost significant resolution of the cervical emphysema and pneumomediastinum confirmed by daily chest X-ray and control thorax CT (Figures 3 and 4). When the symptomes of patient disappeared, he was discharged home after 6 days.

\section{Discussion}

Trauma is one of the major reasons among the patients with pneumomediastinum. Following spontaneous pneumothorax it is rarely seen in young patients as a consequence of peripheral alveolar rupture, severe cough, 


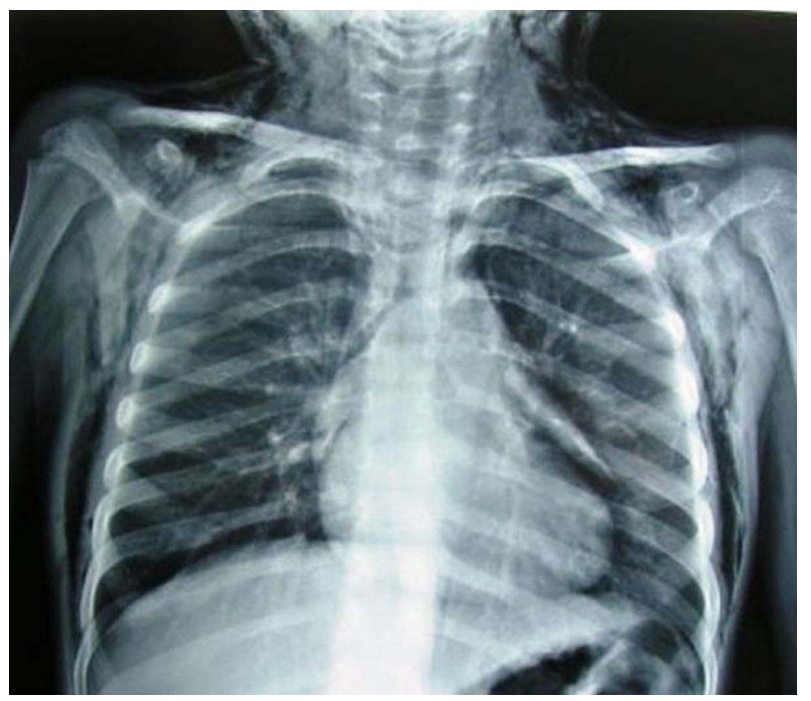

Figure 1. PA chest radiograph on the first admission; demonstrates subcutaneous emphysema both in cervical and thoracic regions.

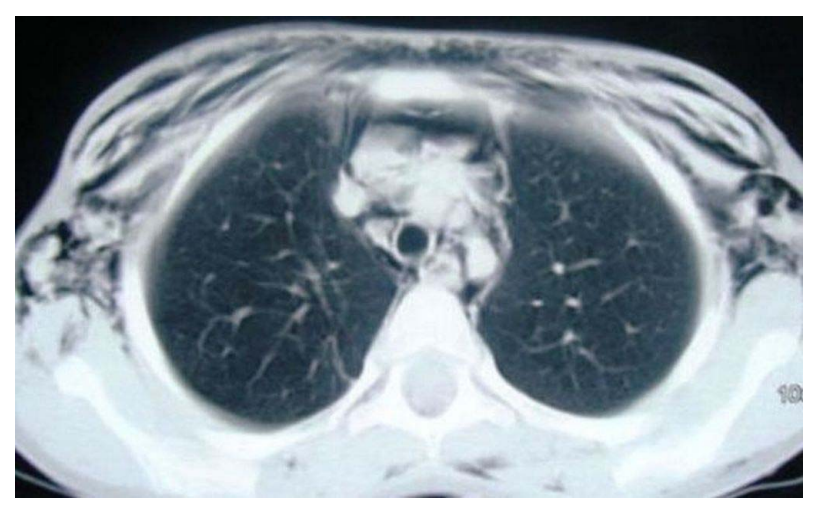

Figure 2. Torax CT on the first admission; reveals pneumomediastinum and subcutaneous emphysema.

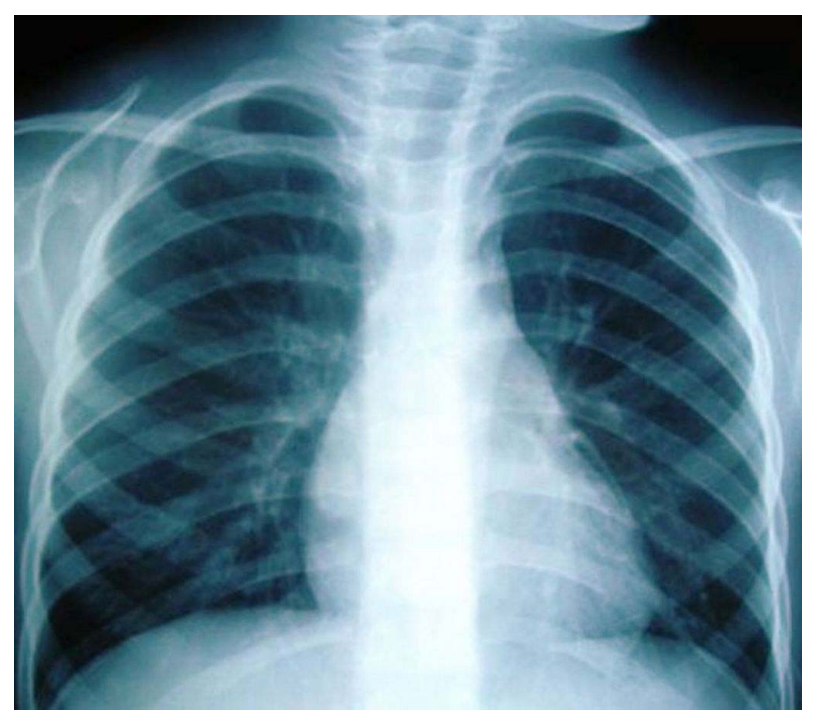

Figure 3. PA chest radiograph resolution of pneumomediastinum after treatment.

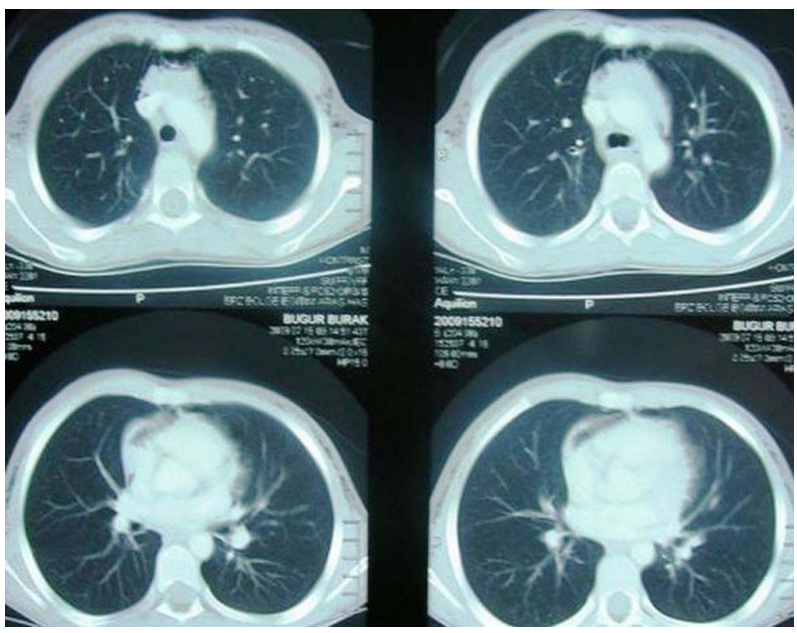

Figure 4. Torax CT shows spontaneous resolution of pneumomediastinum after nonsurgical.

heavy exercise and intraabdominal high pressure can cause pneumomediastinum [1]. Other rare predisposing factors include bronchial astma, voluntary valsalva manoeuvres during smoking of marijuana or cocaine, diving and vaginal delivery $[4,5]$.

Over distention of intact alveolus is called barotrauma. Accumulation of air in the intertisium as a result of alveolar and alveolar septal rupture reaches the neck along the perivascular and peribronchial area. Barotrauma can cause mucosal perforation on upper airway too. High air pressure affects both subcutanaeus tissue of head and neck and also indirectly affects mediastinum.

After dental operation, tonsillectomy, tracheastomy, head and neck surgery, cranio-facial trauma, high positive pressure ventilation or variations in aircraft cabin pressure, pneumomediastinum with subcutaneous emphysema is reported in literature. Explosion of bottle in mouth and inflation of bicycle and tractor tyre are reported as etiologic factors which causes orapharengeal barotraumas [6,7].

Characteristic sign and symptomes are dispnea, subcutaneous emphysema, muffled heart sounds, a loud crunching sound over the precordium synchronous with the heart sounds pneumothorax, mediastinal pressure symptoms (dyspnea, cyanosis, plump veins and circulatory failure) and radiological evidence of air in the mediastinum.

Rapid diagnosis is important because it is a potentially lethal situation. Besides tension and/or bilateral pneumothorax, serious complications were reported as tension pneumomediastinum cause cardiac compression and decreased cardiac output. Pneumomediastinum patients should be monitored closely for avoiding these problems. Spontaneous resolution is expected for patients with non-complicated pneumomediastinum by avoiding analgesics and the Valsalva maneuver. For severe complica- 
tions, mediastinal needle aspiration, cervical mediastinoscopy, tracheostomy or emergency thoracotomy methods may be used.

W. G. Bowsher et al. and Joon-Kyoo Lee and Sang-chul Lim's have been reported faryngo-esophageal perforations after orapharengeal barotrauma caused by the explosion of bottles in mouth. Accordingly, the cervical emphysema, pneumomediastinum have been also reported. Conservative treatment approaches have been primarily used based on the patient's clinic. In some cases, surgical drainage and primary repair was applied $[6,8]$.

Patients with pneumomediastinum have high mortality and morbidity rate and diagnosed with clinical examination, chest X-ray or computed tomography of the chest. Our case was diagnosed by clinical examination and supported by radiological examinations. Depending on the etiology, antibiotics should be given to patients with pneumomediastinum for preventing mediastinitis [9]. Because of the possibility of complications, all patients with pneumomediastinum such a period of time (at least 24 hours) should be observed in the hospital. Pneumomediastinum usually disappear within a week's time $[3,10]$.

For the treatment of severe subcutaneous emphysema microdrainage is needed. [10]. Leo et al. reported that in their 1008 major thoracic operation, 11 patients with severe subcutaneous emphysema were needed microdrainage (1.1\%). The procedure was reported to be effective and free of complications [10]. The successful microdrainage of the subcutaneous emphysema with simply constructed angiocatheters was first described by Beck et al. [11]. This was followed by three other adult case reports [12-14]

$\mathrm{O}_{2}(4 \mathrm{l} / \mathrm{min})$ therapy and 2nd generation cephalosporin for infection prophylaxis were given to patient. At the end of first 48 hours of the patient followed up for six days in the hospital, clinical and radiological improvement were observed in pneumomediastinum and subcutaneous emphysema, an additional surgical and interventional procedure were not needed.

\section{Summary}

Altough pneumomediastinum with subcutaneous emphysema after mechanical ventilation is frequently reported in literature, pneumumediastinum caused by orapharengeal barotrauma is rarely reported. We presented a case of oropharyngeal barotrauma-induced pneumomediastinum treated with conservative approach in order to provide contribution to the literature.

\section{REFERENCES}

[1] A. Yellin, M. Gapany-Gapanavicius and Y. Lieberman, "Spontaneous Pneumomediastinum: Is It a Rare Cause of
Chest Pain?” Thorax, Vol. 38, No. 5, 1983, pp. 383-385. doi:10.1136/thx.38.5.383

[2] J. B. Jougon, M. Ballester, F. Delcambre, T. Mac Bride, C. E. Dromer and J. F. Velly, "Assessment of Spontaneous Pneumomediastinum: Experience with 12 Patients,” The Annals of Thoracic Surgery, Vol. 75, No. 6, 2003, pp. 1711-1714. doi:10.1016/S0003-4975(03)00027-4

[3] A. E. Newcomb and C. P. Clarke, "Spontaneous Pneumomediastinum: A Benign Curiosity or a Significant Problem?” Chest, Vol. 128, No. 5, 2005, pp. 3298-3302.

[4] G. J. Koullias, D. P. Korkolis, X. J. Wang and G. L. Hammond, "Current Assessment and Management of Spontaneous Pneumomediastinum: Experience in 24 Adult Patients,” European Journal Cardio-Thoracic Surgery, Vol. 25, No. 5, 2004, pp. 852-855. doi:10.1016/j.ejcts.2004.01.042

[5] F. W. Sutherland, S. Y. Ho and C. Campanella, "Pneumomediastinum during Spontaneous Vaginal Delivery,” The Annals of Thoracic Surgery, Vol. 73, No. 1, 2002, pp. 314-315. doi:10.1016/S0003-4975(01)02729-1

[6] W. G. Bowsher and G. S. Kenyon, “Accidental Oropharyngeal Injury,” British Medical Journal (Clinical Research Edition), Vol. 284, No. 6331, 1982, p. 1752. doi:10.1136/bmj.284.6331.1752

[7] M. Kraus, J. Peiser, N. Bartal and D. M. Fliss, "Cervical Subcutaneous Emphysema Due to Oropharyngeal Barotrauma," Journal of Oral and Maxillofacial Surgery, Vol. 53, No. 10, 1995, pp. 1215-1217. doi:10.1016/0278-2391(95)90639-8

[8] J.-K. Lee and S.-C. Lim, "Barotraumatic Perforation of Pharyngoesophagus by Explosion of a Bottle into the Mouth,” Yonsei Medical Journal, Vol. 46, No. 5, 2005, pp. 724-728. doi:10.3349/ymj.2005.46.5.724

[9] H. Miura, O. Taira, S. Hiraguri, K. Ohtani and H. Kato, "Clinical Features of Medical Pneumomediastinum," Annals of Thoracic Cardiovascular Surgery, Vol. 9, No. 3, 2003, pp. 188-191.

[10] F. Leo, P. Solli, G. Veronesi, et al., "Efficacy of Microdrainage in Severe Subcutaneous Emphysema," Chest, Vol. 122, No. 4, 2002, pp. 1498-1499. doi:10.1378/chest.122.4.1498-a

[11] P. L. Beck, S. J. Heitman and C. H. Mody, "Simple Construction of a Subcutaneous Catheter for Treatment of Severe Subcutaneous Emphysema,” Chest, Vol. 121, No. 2, 2002, pp. 647-649. doi:10.1378/chest.121.2.647

[12] M. Ozdogan, A. Gurer, A. K. Gokakin, S. Gogkus, I. Gomceli and R. Aydin, "Treatment of Severe Subcutaneous Emphysema by Fenestrated Angiocatheter,” Intensive Care Medicine, Vol. 31, No. 1, 2005, p. 168. doi:10.1007/s00134-004-2443-X

[13] L. A. Perkins and S. F. Jones, "Resolution of Subcutaneous Emphysema with Placement of Subcutaneous Fenestrated Angiocatheter," Respiratory Medicine Extra, Vol. 3, No. 3, 2007, pp. 102-104. doi:10.1016/j.rmedx.2007.05.001

[14] R. Srinivas, N. Singh, R. Agarwal and A. N. Aggarwal, "Management of Extensive Subcutaneous Emphysema and Pneumomediastinum by Micro-Drainage: Time for a Rethink?” Singapore Medical Journal, Vol. 48, No. 12, 2007, pp. e323-e326. 